\title{
Browsing Proof Markup Language Provenance: Enhancing the Experience
}

\author{
Nicholas Del Rio, Paulo Pinheiro da Silva, and Hugo Porras \\ The University of Texas at El Paso, Computer Science, \\ 500 W. University Ave. El Paso TX 79968 USA
}

\section{Introduction}

Probe-It! is a browser that allows users to navigate through Proof Markup Language (PML) based provenance traces by interacting with a number of different perspectives or views [1]. These views provide specific renderings or presentations for the different kinds of provenance information defined in the PML ontology [2]. Throughout our three year experience with Probe-It! we have gathered requirements from users who have a need for browsing PML captured from theorem provers in the Thousands of Problems for Theorem Provers (TPTP) and Homeland Security domains as well as from scientific processes in areas such as solar astronomy, seismology, and environmental science. This paper briefly describes the enhancements made to Probe-It! to improve usability and performance with regards to visualization.

\section{Usability}

Probe-It!'s global justification view grants users visual access to a PML based provenance trace in its entirety. The global justification view renders PML justifications as a DAG, where nodesets are represented as graph nodes and the hasAntecedent relationships are represented by the arcs connecting nodesets. The nodesets composing the proof DAG are each represented as a box that contains a nodeset's conclusion. These DAGs have the potential to be very large, especially in the Thousands of Problems for Theorem Provers (TPTP) domain, where many of the proofs consist of hundreds of nodesets. In these cases providing users with options on how to navigate around these large provenance traces becomes essential. In fact, it was noted by a member of the TPTP community that his instinct was to "drag" around the global view justification canvas, which Probe-It! was not capable of. Probe-It! now addresses this limitation by now supporting Google Earth-like navigation capabilities rather than relying solely on a scrollable pane to move the DAG around. In addition users now have the ability to zoom in/out, abstract the information shown in the nodesets, a feature found in [4, or move around the graph by dragging on the canvas or using the panner. 
Another enhancement to Probe-It!, that we hope will increase the quality of users' experience, was the adoption of the Inference Web [3] group's local view. The local view presents both data transformation trace information (PML-J) and transformation trace metadata (PMP-J) associated with a single nodeset, where as the global justification view hides much of the PML-P information and puts more emphasis on presenting the structure of a justification as a whole. The information presented to users in the local view is categorized by: the selected nodeset's conclusion, how the selected nodeset was derived (antecedents, rule), what its conclusion is used to derive (consequents of), and what it is used to finally derive (final conclusion of proof). It is also possible to navigate to any other nodesets that were used to derive or are derived from the currently selected nodeset, through the use of links. Upon selection of new nodeset, the information presented in the local view is updated to reflect the newly selected nodeset. We are currently devising scenarios in which to measure the effectiveness of the local view in a scientific context, where nodeset conclusions are visualizable datasets rather than logical statements.

\section{Performance: Preprocessed Views}

In cases where PML traces are documenting derivations of scientific products rather than derivations of logical conclusions, Probe-It! may be required to transform nodeset conclusions captured in their raw form into a complex visualization that a scientist is familiar with or expecting. Because performing these transformations in viewing time can really slow down the global justification browsing experience, we have employed a preprocessing system that caches any visualization that can be generated from nodeset conclusions. In fact, as soon as any PML nodeset is written to our file system, the cacher can be initiated and thus can be run in parallel with the execution of the workflow being audited.

The importance of such a caching system was first realized when we attempted to browse the PML capturing a run of Hole's code (an iterative process used for modeling the Earth's crust by seismologists) in the global view. In this case, Probe-It! was required to generate a Visualization Toolkit (VTK) based visualization for each of the 300 nodesets composing the trace. From our experiences, we have learned that seismologists prefer to inspect Hole's code datasets visually, and so it is not useful for them to have access to provenance when verifying if their models have converged, if they cannot visualize them.

\section{References}

1. del Rio, N., da Silva, P.P.: Probe-it! visualization support for provenance. In: Proceedings of the Second International Symposium on Visual Computing (ISVC 2), Lake Tahoe, NV, pp. 732-741. Springer, Heidelberg (2007)

2. McGuinness, D., Ding, L., da Silva, P.P., Chang, C.: PML2: A Modular Explanation Interlingua. In: Proceedings of the AAAI 2007 Workshop on Explanation-aware Computing, Vancouver, British Columbia, Canada, July 22-23 (2007) 
3. McGuinness, D.L., da Silva, P.P.: Inference Web: Portable and Sharable Explanations for Question Answering. In: Proc. of the AAAI Spring Symposium Workshop on New Directions for Question Answering, Stanford, CA, USA, March 2003, pp. 67-71. AAAI Press, Menlo Park (2003)

4. Trac, S., Puzis, Y., Sutcliffe, G.: An interactive derivation viewer. In: Proceedings of the 7th Workshop on Workshop on User Interfaces for Theorem Provers, 3rd International Joint Conference on Automated Reasoning. Electronic Notes in Theoretical Computer Science, vol. 174, pp. 109-123 (2006) 